\title{
A Fundamental Tradeoff between Performance and Sensitivity within Haptic Rendering
}

\author{
Paul G. Griffiths \\ Dept. of Mechanical Engineering \\ University of Michigan \\ Ann Arbor, MI 48109 \\ Email: paulgrif@umich.edu
}

\author{
R. Brent Gillespie \\ Dept. of Mechanical Engineering \\ University of Michigan \\ Ann Arbor, MI 48109 \\ Email: brentg@umich.edu
}

\author{
Jim S. Freudenberg \\ Dept. of Electrical Engineering \\ \& Computer Science \\ University of Michigan \\ Ann Arbor, MI 48109 \\ Email: jfr@eecs.umich.edu
}

\begin{abstract}
In this paper we show that for haptic rendering using position feedback, the structure of the feedback loop imposes a fundamental tradeoff between accurate rendering of virtual environments and sensitivity of closed-loop responses to hardware variations and uncertainty. Due to this tradeoff, any feedback design that achieves high-fidelity rendering incurs a quantifiable cost in terms of sensitivity. Analysis of the tradeoff reveals certain combinations of virtual environment and haptic device dynamics for which performance is achieved only by accepting very poor sensitivity. This analysis may be used to show that certain design specifications are feasible and may guide the choice of hardware to mitigate the tradeoff severity. We illustrate the predicted consequences of the tradeoff with an experimental study.
\end{abstract}

\section{INTRODUCTION}

Design of a feedback controller involves compromises between various conflicting objectives. These tradeoffs are imposed by factors external to the controller design such as the location of sensors and actuators, and limitations of the hardware such as sample-rate, delay, bandwidth, and quantization. By quantifying these tradeoffs we reveal certain relationships between hardware and feedback properties which are satisfied for all controller designs. Such knowledge allows one, for instance, to identify infeasible specifications before any feedback design is attempted. If design specifications cannot be relaxed, interpretation of the underlying mathematical relationships can provide guidance in selecting different hardware to reduce the severity of tradeoffs. Furthermore, if feasible feedback designs do exist, knowledge of fundamental tradeoffs can confirm that a particular design strikes a favorable compromise between conflicting goals.

Feedback control in haptic rendering is used to shape the closed-loop dynamics of a haptic device to match the dynamics of a desired virtual environment. This use of feedback, however, must be weighed against the costs associated with feedback, notably the potential for instability. Previous work in haptic interface systems has addressed tradeoffs between performance and stability due to sampling [1]-[9], quantization [1], [5], [6], [8], [9], hardware damping [1]-[3], [5]-[9], and nonlinearities [1], [2], [4]-[9]. An important characteristic of feedback design which has not been analyzed for haptic rendering is the sensitivity of the closed-loop response to parameter variations in the hardware and model uncertainty.
Predictions about stability or performance become less reliable as the sensitivity to hardware dynamics increases, and although feedback may be used attenuate sensitivity, feedback may also amplify sensitivity.

Tradeoffs between performance and sensitivity are well characterized for typical servo-control applications [12]; however feedback design for haptic interface cannot be treated as a typical servo-control problem. An important goal of feedback in servo-control applications is to reject disturbances that enter at the actuator input. In haptic rendering with backdrivable devices, the operator's input enters at the actuator input and rejecting this input is not the goal of feedback. Instead the virtual environment provides the desired response to the human operator. Feedback design for haptic rendering may then be treated as a model-matching problem which is not solved by standard loop-shaping techniques. A different control strategy is required for haptic rendering, and additional design tradeoffs exist which have no counterpart in servocontrol systems.

The Bode sensitivity function characterizes multiple important properties of a feedback system including stability robustness and sensitivity of closed-loop transfer functions to variations in the hardware dynamics [12]. In typical servocontrol problems, the Bode sensitivity function also describes the disturbance response of performance outputs; however, for a certain class of feedback systems, attenuation of this disturbance response is not achieved by attenuating the Bode sensitivity function. This class of systems is characterized by performance outputs that differ from the measured outputs and disturbance inputs that affect the plant through different dynamics than the control inputs. As detailed in Freudenberg et al. [13], an algebraic (frequency-by-frequency) tradeoff exists within these systems between performance goals and feedback properties described by the Bode sensitivity function.

In this paper we apply the results of [13] to reveal a tradeoff between performance and sensitivity not previously analyzed within haptic interface systems. Our analysis shows that, at a frequency, all feedback designs must compromise between rendering the virtual environment accurately and reducing sensitivity to haptic device dynamics. We capture the severity of this tradeoff in a single frequency-dependent parameter that depends on the virtual environment and haptic 
device dynamics. Based on the tradeoff, the cost in terms sensitivity to render a virtual environment accurately is independent of the feedback design, and is large at frequencies where inherent dynamics of the haptic device would mask the virtual environment dynamics. We introduce a controller design that cancels the haptic device dynamics as needed and is subject to the attendant poor sensitivity for certain virtual environments. Due to the tradeoff, the only way to reduce sensitivity without sacrificing performance is to re-design the haptic device hardware with reduced inherent dynamics such as smaller inertia and damping.

The tradeoff we show is fundamental to the hardware and does not depend on the controller implementation or complexity. Our analysis assumes a haptic device, equipped with position sensing, whose dynamic response to the control input is the same as the dynamic response to the human operator's applied force. For simplicity, we treat single-axis haptic devices. We work with continuous linear time-invariant models of the haptic interface system and do not capture sampleddata effects, quantization, and other nonlinearities. While these factors present their own limitations and tradeoffs, the tradeoff we discuss exists in addition to these, and its severity cannot be diminished by increasing sample rate, improving sensor quantization, or minimizing nonlinear dynamics.

\section{RENDERING VIRTUAL ENVIRONMENTS USING Position FEEDBACK CONTROL}

\section{A. Hardware \& Controller}

In a standard configuration of haptic rendering, a human operator grasps and applies forces to a motorized, computercontrolled manipulator. Figure 1 shows this standard setup using a direct-drive, single-axis, rotary handwheel equipped with an encoder for position measurement. The controller reads the handwheel position $X$, as measured by the encoder, and computes a motor command. The amplified motor command drives a torque $u$ that acts on the handwheel. The human operator also applies a torque $F$ on the handwheel, which affects the position $X$ through the same dynamics as $u$. The motor, amplifier, encoder, and handwheel together comprise the haptic device. The haptic device may be linear or rotary, and we henceforth refer to $F$ and $u$ as forces rather than torques without loss of generality.

We assume a linear systems framework, where variables are defined by the Laplace transform of their respective signals and transfer functions are defined by the Laplace transform of their respective operators. Let us capture the haptic device dynamics in the transfer function $P$. Then the position of the haptic device is

$$
X=P(F+u) .
$$

The controller describes the transfer function from position measurement to motor command. We capture the controller dynamics in the transfer function $C$. Then the motor command is given by

$$
u=-C X
$$

The human operator perceives the closed-loop response from $F$ to $X$. Closing the loop between $P$ and $C$, we find that this response is $P /(1+P C)$.

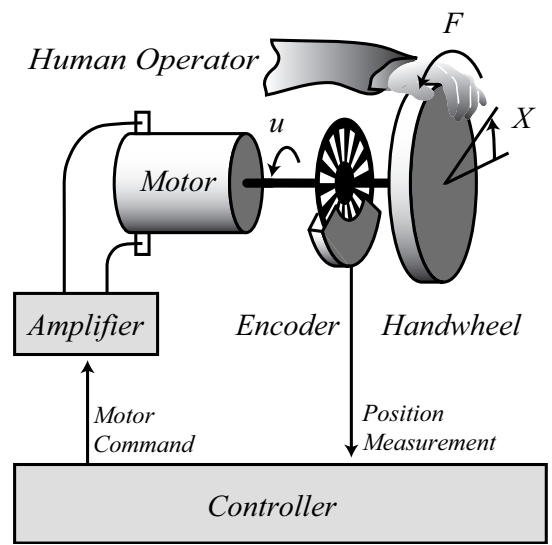

Fig. 1. Schematic of the haptic interface system hardware and controller.

\section{B. Posing feedback design as a model-matching problem}

The purpose of feedback control in haptic rendering is to shape the closed-loop response of the haptic device position $X$ to the human operator's input $F$. The desired response is generated by the virtual environment dynamics, denoted by the transfer function $R_{d}$. Let us define $X_{d}$ to be the desired closed-loop response of $X$ to $F$. Then

$$
X_{d}=R_{d} F
$$

and accurate rendering of the environment dynamics is achieved by attenuating the response of the error signal $X-X_{d}$ to the human operator force $F$.

We use the standard form of the general control configuration [12] to capture the feedback design problem of haptic rendering. As shown in Fig. 2, the standard form consists of a generalized multivariable plant $G$ in feedback with a generalized controller $K$. The generalized plant $G$ describes the input/output responses from disturbance inputs $w$ and control inputs $u$ to performance outputs $z$ and measured outputs $y$ :

$$
\left[\begin{array}{l}
z \\
y
\end{array}\right]=\left[\begin{array}{ll}
G_{z w} & G_{z u} \\
G_{y w} & G_{y u}
\end{array}\right]\left[\begin{array}{l}
w \\
u
\end{array}\right] .
$$

We denote the closed-loop disturbance response from $w$ to $z$ by $T_{z w}$. If all signals are scalar, the disturbance response is given by

$$
T_{z w}=G_{z w}+G_{z u} K\left(1-G_{y u} K\right)^{-1} G_{y w} .
$$

Performance goals are achieved by designing the generalized controller $K$ to attenuate the disturbance response $T_{z w}$.

The block diagram shown in Fig. 3 depicts the feedback design of haptic interface posed in the general control configuration. The exogenous input $w$ is generated by the human operator force $F$, and performance output $z$ is defined by the normalized error signal

$$
z \triangleq R_{d}^{-1}\left(X-X_{d}\right)
$$




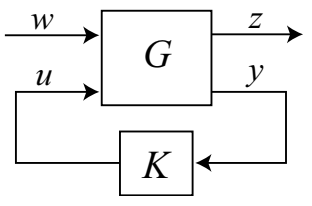

Fig. 2. Block diagram of the general control configuration.

The generalized control input $u$ is synonymous with the motor torque $u$, and the measured output $y$ is simply $X$. Then the elements of the generalized plant $G$ (contained within the dashed box in Fig. 3) are

$$
G=\left[\begin{array}{cc}
P / R_{d}-1 & P / R_{d} \\
P & P
\end{array}\right] .
$$

We may compute the disturbance response $T_{z w}$ by substituting (7) into (5) and $-C$ for $K$ :

$$
T_{z w}=\frac{1}{R_{d}}\left(\frac{P}{1+P C}-R_{d}\right) .
$$

Note that the term $P /(1+P C)$ describes the actual closed-loop response of $X$ to the human operator input $F$. We refer to this transfer function as the rendered virtual environment, which we denote by

$$
R \triangleq \frac{P}{1+P C} .
$$

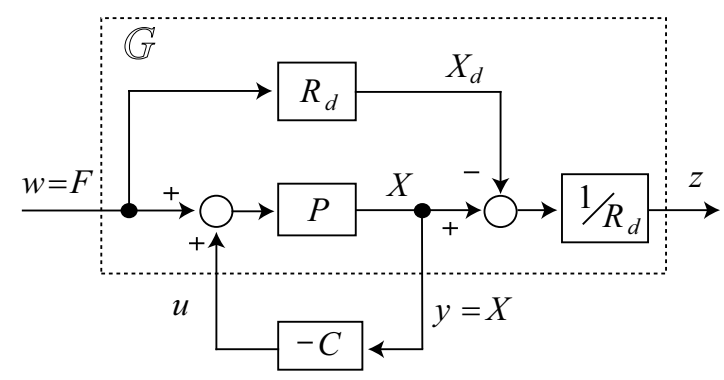

Fig. 3. A model-matching block diagram of haptic rendering. The human operator feels the rendered virtual environment $R$, the response $X / F$; whereas the desired response is given by virtual environment $R_{d}$. The performance variable $z$ measures distortion, the normalized error between the actual and desired responses.

The disturbance attenuation problem captured by (8) may be treated as a model-matching problem. To attenuate the disturbance response $T_{z w}$, we must design $C$ to reduce the mismatch between rendered virtual environment $R$ and the desired virtual environment $R_{d}$. We denote the closed-loop disturbance response (8) for haptic rendering by $\Theta_{c}$ and call this relative error between $R$ and $R_{d}$ distortion. Exact modelmatching $R \equiv R_{d}$, known as perfect transparency [14], is achieved when $\Theta_{c} \equiv 0$.

We note that the benefit of feedback in reducing closedloop distortion should be quantified relative to the distortion of the open-loop system. We denote distortion of the openloop system by $\Theta_{o} \triangleq\left(P-R_{d}\right) / R_{d}$. Distortion of the open-loop system may be smaller than closed-loop distortion at some frequencies implying a cost rather than benefit of feedback.

\section{Sensitivity to parameter variations in the haptic device}

In addition to nominal performance, any practical feedback design must provide a degree of robustness to variations in the haptic device. The Bode sensitivity function $S$ describes several important feedback properties including the sensitivity of closed-loop transfer functions to variations in hardware dynamics [12]. In terms of the haptic device dynamics $P$ and the controller $C$, the Bode sensitivity function is

$$
S=\frac{1}{1+P C} .
$$

Let us then consider the sensitivity of the rendered virtual environment $R$ to the haptic device dynamics $P$. One may show that

$$
\frac{P}{R} \frac{d R}{d P}=S .
$$

Thus $S$ describes the differential change in the rendered environment $d R / R$ to a differential change in the haptic device model $d P / P$. Then, to a first-order approximation, relative error in the haptic device dynamics results in a relative error between the nominal and actual rendered virtual environment scaled by the Bode sensitivity function $S$. To reduce the sensitivity of $R$ to variations in the haptic device dynamics and model uncertainty, the feedback design must attenuate $S$.

\section{Structure of the haptic interface controller}

The haptic interface controller is typically partitioned into two parts: a simulation of the virtual environment and a virtual coupler [15]. This latter element connects the hardware with the virtual environment. As shown in Fig. 4, the virtual coupler produces an input $F_{e}$ to the virtual environment and receives the desired position of the haptic device $X_{d}$. Partitioned in this way, design of the controller is split into two problems: creating an accurate simulation of the virtual environment dynamics, and designing a generalized virtual coupler to render that virtual environment with low distortion. With this structure, various virtual environments can, in theory, be interchanged without redesigning the virtual coupler. Note that for haptic applications involving scaling between the haptic device and the virtual environment, we assume without loss of generality in our analysis that these scaling factors are internal to the virtual environment.

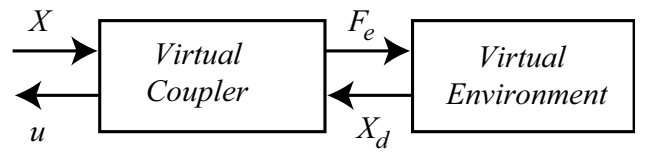

Fig. 4. Architecture of the controller $C$, which describes the transfer function from $X$ to $u$.

We note that the virtual coupler may be modeled after mechanical elements such as springs and dampers, but can more generally be described by four transfer functions relating 
the two inputs and two outputs. In Section IV we find design directives for these four transfer functions which minimize distortion. For now we defer discussion about design of the virtual coupler, because the tradeoff between performance and sensitivity, which is the focus of the present work, is independent of the structure imposed on $C$.

\section{A Tradeoff Between Performance and SENSITIVITY}

\section{A. Background}

For many common feedback systems such as servo-control applications, the disturbance response $T_{z w}$ is described by the Bode sensitivity function $S$. However, within multivariable control systems where (a) the output and performance variables differ, and (b) the control and exogenous input affect the plant through different dynamics, $S$ does not describe $T_{z w}$. Then, at a frequency, the feedback design cannot attenuate both the disturbance response and sensitivity as dictated by an algebraic (frequency-by-frequency) identity presented in [13]. We now briefly reproduce this identity in terms of the general control configuration.

Recall that according to (5), the disturbance response $T_{z w}$ in terms of the elements of the generalized plant $G$ is $G_{z w}+G_{z u} K\left(1-G_{y u} K\right)^{-1} G_{y w}$. The Bode sensitivity function for the general control configuration is $S=1 /\left(1-G_{y u} K\right)$. Combining the expressions for $T_{z w}$ and $S$ we can find an expression that does not depend explicitly on the controller $K$. Let $\Gamma \triangleq G_{z u} G_{y w} /\left(G_{z w} G_{y u}\right)$. Then we have the algebraic identity

$$
\frac{T_{z w}}{G_{z w}}=1+\Gamma(S-1) .
$$

Only when $\Gamma=1$ is disturbance attenuation described by the Bode sensitivity function. There are two special cases where $\Gamma \equiv 1$ : (a) systems whose performance variable is also the measured output, that is $G_{z u}=G_{y u}$ and $G_{z w}=G_{y w}$, and (b) systems whose control and exogenous input enter the system through the same dynamics, that is $G_{z u}=G_{z w}$ and $G_{y u}=G_{y w}$.

For systems where $\Gamma \not \equiv 1$, achieving good performance and low sensitivity are competing goals. The severity of the tradeoff is determined by $\Gamma$ and is generally frequency dependent. Recall that we wish to attenuate both $T_{z w}$ and $S$. However, at frequencies where $|\Gamma(j \omega)|<<1$, the cost to attenuate the closed-loop disturbance response relative to the open-loop disturbance response is large amplification of $S(j \omega)$. On the other hand, at frequencies where $|\Gamma(j \omega)|>>1$, the cost to attenuate the Bode sensitivity function is large amplification of the closed-loop disturbance response relative to the openloop disturbance response. We note, furthermore, that in (12) both $S$ and $T_{z w}$ may be large. Indeed, at high-frequencies, both the Bode sensitivity function and the ratio of the closedloop disturbance response to the open-loop disturbance must approach 1 for any proper feedback design.

\section{B. Application to haptic rendering}

We now interpret the tradeoff implied by (12) for haptic rendering with position feedback. We recall that in (12) the closed-loop disturbance response $T_{z w}$ is $\Theta_{c}$ and the open-loop disturbance response $G_{z w}$ is $\Theta_{o}$. Then performance afforded by feedback control is gauged by attenuation of $\Theta_{c} / \Theta_{o}$. We note that without feedback control, the Bode sensitivity function $S$ and the ratio $\Theta_{c} / \Theta_{o}$ are both unity.

The tradeoff severity $\Gamma$ is found by substituting (7) into $G_{z u} G_{y w} /\left(G_{z w} G_{y u}\right)$. Further substituting open-loop distortion $\Theta_{o}$ for $\left(P-R_{d}\right) / R_{d}$, we find that

$$
\Gamma=1+\frac{1}{\Theta_{o}} .
$$

Given a fixed device model $P$, the term $1 / \Theta_{o}$ approaches 0 as $R_{d}$ approaches 0 . Then, for any $R_{d} \not \equiv 0$, the tradeoff severity $\Gamma \not \equiv 1$ and there exists a tradeoff between attenuating distortion and attenuating the Bode sensitivity function. The tradeoff is most severe at frequencies where $\Theta_{o} \rightarrow-1$ or frequencies where $\Theta_{o} \rightarrow 0$.

We first consider the situation where $\Theta_{o} \rightarrow-1$ and $\Gamma \rightarrow 0$. From the definition of $\Theta_{o}$, we see that $\Theta_{o} \rightarrow-1$ at frequencies where $\left|R_{d}(j \omega)\right|>>|P(j \omega)|$. At these frequencies, the magnitude of the desired closed-loop response given by the virtual environment $R_{d}$ is much greater than the response of the haptic device dynamics $P$, and partial cancellation of the device dynamics $P$ is required. The cost of partially cancelling device dynamics is large amplification of the Bode sensitivity function.

Let us alternatively consider the situation where $\Theta_{o} \rightarrow 0$ and $\Gamma \rightarrow \infty$. This situation arises as the desired virtual environment $R_{d}$ approaches the open-loop dynamics of the haptic device $P$. Little or no feedback control is required to achieve low distortion since open-loop distortion is already nearly 0 . However, the Bode sensitivity function approaches 1 as the feedback gain approaches 0 . We may use feedback to attenuate sensitivity, but only by accepting large amplification of the ratio $\Theta_{c} / \Theta_{o}$.

An important consequence of (12) is that, at frequencies where $\Gamma \neq 1$, any feedback design that attenuates the ratio $\Theta_{c} / \Theta_{o}$ cannot also attenuate the Bode sensitivity function $S$. Furthermore, regardless of the controller synthesis technique or controller complexity, $S \rightarrow 1-1 / \Gamma$ at frequencies where $\Theta_{c} / \Theta_{o} \rightarrow 0$. Substituting (13) for $\Gamma$, we reduce this limit to

$$
S \rightarrow \frac{R_{d}}{P} .
$$

At frequencies where $\left|R_{d}(j \omega)\right| /|P(j \omega)|$ is large, accurate rendering of the virtual environment can only be achieved by accepting very poor robustness to variations in the haptic device dynamics.

The virtual environment $R_{d}$ and haptic device model $P$ are transfer functions from force to motion; thus a large magnitude of either transfer function corresponds to a small mechanical impedance. It is then not surprising that, as given by (14), poor sensitivity results when we accurately render a virtual 
environment with a small mechanical impedance relative to the mechanical impedance of the haptic device. We note, however, that this sensitivity is not that typically recognized in feedforward control-the controller $C$ is indeed in feedback with the haptic device. Furthermore, the sensitivity to hardware dynamics induced by feedback control may be much greater than the unity sensitivity of feed-forward control. Perhaps less intuitive is the frequency dependent nature of the tradeoff. For instance, rendering a pure spring with low distortion using a haptic device with inertia induces sensitivity that increases with frequency.

\section{Virtual Coupler Design}

The design tradeoff introduced above predicts the cost to achieve low distortion for any feedback design. With this analysis one may evaluate whether a feedback design efficiently trades off performance for sensitivity; however, the analysis does not provide a design technique. We now discuss design of the virtual coupler introduced in Section II-D. Our approach is to find a parameterization of the virtual coupler design which provides useful terms for tuning the closed-loop distortion. We then generate design directives for optimizing performance, and use such a design in Section $\mathrm{V}$ to demonstrate experimentally the sensitivity induced as the distortion is reduced. We note, however, that this design optimized for performance may not necessarily be appropriate. While it highlights one point in the tradeoff between performance and sensitivity, one may choose other points in the tradeoff which provide a different balance of feedback properties.

\section{A. Performance of the generalized virtual coupler}

As discussed in Section II-D, the haptic interface feedback controller $C$ is typically partitioned into the virtual environment and virtual coupler. The virtual coupler is generally fixed and should accommodate a range of virtual environments. Referring to Fig. 4, the virtual coupler describes the response from the haptic device position $X$ and desired position $X_{d}$ to the motor command $u$ and the virtual environment force $F_{e}$. This set of input/output responses is often modeled after a physical system such as a spring [15]. Let us simply express these input/output relationships by the matrix of transfer functions $B \triangleq\left[\begin{array}{ll}B_{11} & B_{12} \\ B_{21} & B_{22}\end{array}\right]$ such that

$$
\left[\begin{array}{c}
u \\
F_{e}
\end{array}\right]=B\left[\begin{array}{c}
X \\
X_{d}
\end{array}\right]
$$

Figure 5 shows the block diagram of the haptic interface system with $B$ interposed between the virtual environment and haptic device. We note that $B$ is more general than the virtual coupler described in [1] or extended in [15]. Each controller element of $B$ may be any transfer function, and we impose no relationships between elements. We refer to $B$ as a generalized virtual coupler.

To focus on the design of $B$, we find it useful to remove the virtual environment from the problem and just consider the feedback interconnection of the haptic device $P$ with

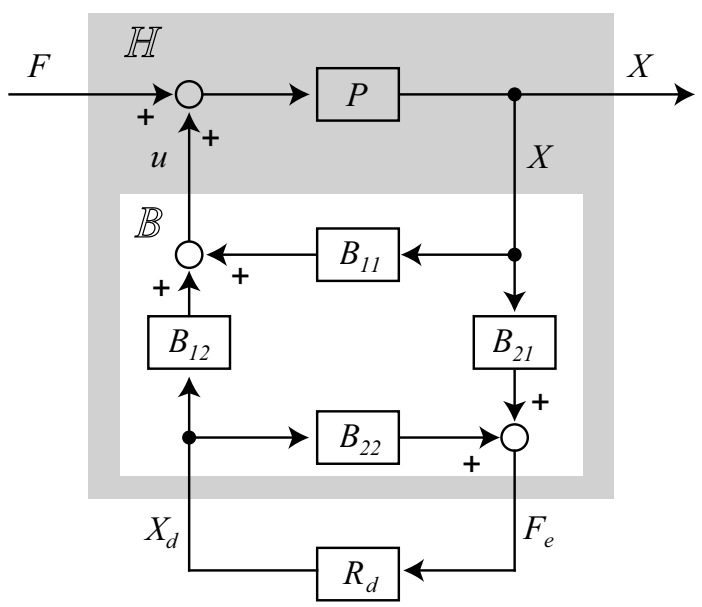

Fig. 5. Block diagram with the controller partitioned into a generalized virtual coupler $B$ and virtual environment $R_{d}$. Design directives may be developed for $B$ by considering the multivariable response of $H$.

the generalized virtual coupler $B$. Referring to Fig. 5, the input/output response of $P$ in feedback with the $B$ is

$$
\left[\begin{array}{c}
X \\
F_{e}
\end{array}\right]=\left[\begin{array}{cc}
\frac{P}{1-B_{11} P} & \frac{B_{12} P}{1-B_{11} P} \\
\frac{B_{21} P}{1-B_{11} P} & B_{22}+\frac{B_{21} B_{12} P}{1-B_{11} P}
\end{array}\right]\left[\begin{array}{c}
F \\
X_{d}
\end{array}\right] .
$$

As we will show, the terms of the four input-output responses of (16) are easily related to closed-loop distortion when $R_{d}$ is reconnected to the virtual coupler.

Let us denote the matrix of four transfer functions in (16) by $H$ in the form of the hybrid matrix (where our mapping is between force and position rather than force and velocity):

$$
\left[\begin{array}{l}
X \\
F_{e}
\end{array}\right]=\left[\begin{array}{ll}
H_{11} & H_{12} \\
H_{21} & H_{22}
\end{array}\right]\left[\begin{array}{l}
F_{h} \\
X_{d}
\end{array}\right] .
$$

The multivariable responses of $H$ are indicated by the dark box in Fig. 5. We note that, if $H_{11} \not \equiv 0$, then $H$ uniquely determines the generalized virtual coupler $B$ according to

$$
\left[\begin{array}{ll}
B_{11} & B_{12} \\
B_{21} & B_{22}
\end{array}\right]=\left[\begin{array}{cc}
\frac{H_{11}-P}{P H_{11}} & \frac{H_{12}}{H_{11}} \\
\frac{H_{21}}{H_{11}} & H_{22}-\frac{H_{12} H_{21}}{H_{11}}
\end{array}\right] .
$$

We then use $H$ as a re-parameterization of the generalized virtual coupler $B$.

Before we connect the virtual environment to $H$, let us remark on the role of the elements of $H$. Referring to (17), the response from the desired position of the haptic device $X_{d}$ to the actual position $X$ is described by $H_{12}$. Clearly, for small error between $X$ and $X_{d}, H_{12}$ must be close to 1 . The virtual environment describes the desired response of $X_{d}$ to the human operator force $F$; however, referring to Fig. 5, we see that the virtual environment generates $X_{d}$ in response to $F_{e}$ not $F$. Thus, to generate the correct desired position $X_{d}$, the term $H_{21}$, which describes the response from $F$ to $F_{e}$, must be close to 1 . The remaining terms $H_{11}$, which describes the 
feed-through from $F$ to $X$, and $H_{22}$, which describes the feedthrough from $X_{d}$ to $F_{e}$, should be attenuated.

Let us now re-introduce the virtual environment in the feedback loop and compute distortion to verify the intuition just developed. Computing the response from $F$ to $X$ in Fig. 5, we find the rendered virtual environment in terms of $R_{d}$ and the elements of $H$ :

$$
R=H_{11}+\frac{H_{12} H_{21} R_{d}}{1-H_{22} R_{d}} .
$$

Recall that closed-loop distortion $\Theta_{c}$ in terms of $R$ is $(R-$ $\left.R_{d}\right) / R_{d}$. Substituting (19) into the expression for $\Theta_{c}$, we find that

$$
\Theta_{c}=\frac{H_{11}}{R_{d}}+\frac{H_{12} H_{21}}{1-H_{22} R_{d}}-1 .
$$

If $H_{11}$ and $H_{22}$ are 0 , and if $H_{12}$ and $H_{21}$ are 1 , then $\Theta_{c}$ is 0 regardless of the virtual environment.

\section{B. Optimizing for performance}

In practice, it is not possible to design the generalized virtual coupler to make distortion small for all environments and across all frequencies. Examining (20), we see that a general strategy to reduce distortion is to attenuate both $H_{11}$ and $\left(H_{12} H_{21}\right) /\left(1-H_{22} R_{d}\right)-1$. To attenuate the latter term, we can select $H_{22}$ such that $\left|H_{22} R_{d}\right|<<1$ and $H_{12} H_{21} \approx 1$. Referring to (16), we see that $H_{22}$ can in fact be made identically zero if $B_{22}=B_{21} B_{12} P /\left(1-B_{11} P\right)$. The cancellation, accomplished by summation rather than inversion, is not in practice difficult using proper elements in $B$. We call generalized virtual couplers with $H_{22} \equiv 0$ cancellation coupler. The term $H_{11}$ which describes the feed-through from $F$ to $X$ can be attenuated at low-frequencies, but due to bandwidth limitations, $H_{11}$ must approach the open-loop dynamics $P$ at high-frequencies. Similarly, $H_{12}$ and $H_{21}$ can be selected to have a response near 1 at low frequencies but must roll-off at high-frequencies.

The closed-loop distortion achieved by the cancellation coupler is tuned by the free parameters $H_{11}, H_{12}$, and $H_{21}$. Through our choice of parameters, we can guarantee that $\Theta_{c}$ satisfies an upper bound for a range of virtual environments. Examining (20), we can upper bound distortion by

$$
\left|\Theta_{c}\right| \leq\left|H_{11}\right| /\left|R_{d}\right|+\left|H_{12} H_{21}-1\right| .
$$

We design the term $\left|H_{12} \mathrm{H}_{21}-1\right|$ and it is independent of the virtual environment. The term $\left|H_{11}\right| /\left|R_{d}\right|$ is upper bounded for any virtual environments satisfies a lower bound.

The design directives we have provided reduce closed-loop distortion but at the cost of other feedback goals such as sensitivity. In the next section, we use a cancellation coupler optimized for performance to highlight the costs associated with achieving low distortion; however one may reasonably choose a different virtual coupler design that accepts some performance penalty to improve sensitivity.

\section{EXPERIMENTAL RESULTS}

We now use a cancellation coupler to render two sample virtual environments experimentally. We tune the feedback design to achieve good performance across as wide a frequency band as possible given our hardware. At frequencies where distortion is low, the Bode sensitivity function must approach the limit $R_{d} / P$. We provide experimental verification of the predicted sensitivity to hardware dynamics by varying the haptic device dynamics.

We render two mass-spring-damper virtual environments, both with a natural frequency of $2 \mathrm{~Hz}$ and a damping ratio of 0.2 , but the gain of the second system is five times the gain of the first. Let $R_{d 1}$ be the first virtual environment, given by

$$
R_{d 1}=\frac{474}{s^{2}+5.03 s+158} \quad(\mathrm{rad} / \mathrm{N}-\mathrm{m}) .
$$

Then let $R_{d 2}$ be the second virtual environment, given by

$$
R_{d 2}=5 R_{d 1} \quad(\mathrm{rad} / \mathrm{N}-\mathrm{m}) .
$$

Values for the mass, damping coefficient, and spring rate of $R_{d 2}$ are $1 / 5$ of those for $R_{d 1}$.

\section{A. Hardware and Controller Design}

We render $R_{d 1}$ and $R_{d 2}$ on a rotary, single-axis, impedancetype haptic device described in [16]. The haptic device dynamics, as determined experimentally, are

$$
P=\frac{1550}{s^{2}+0.775 s} \quad(\mathrm{rad} / \mathrm{N}-\mathrm{m}) .
$$

Figure 6a shows the frequency response of $R_{d 1}$ and $R_{d 2}$ plotted along with the haptic wheel dynamics $P$. For $R_{d 1}$ and $R_{d 2}$ rendered on the haptic wheel, we have the tradeoff severity $\Gamma$ shown in Fig. 6 b.

Design parameters of the cancellation coupler $H_{11}, H_{12}$, and $H_{21}$ are designed according to the design directives given in Section IV. Frequency responses of the parameters are shown in Fig. 7. The magnitudes of $H_{11}$ and $1-H_{12} H_{21}$ are attenuated subject to practical limitations of our hardware such as sampling, quantization, and high-frequency resonances in the haptic device. Digital implementation of the controller is a Tustin discretization at a sampling frequency of $1 \mathrm{kHz}$. The resulting cancellation coupler design achieves the closed-loop distortion shown in Fig. 8, and the Bode sensitivity function for each virtual environment is shown in Fig. 9.

\section{B. Results}

Experimental step responses of the rendered virtual environments are shown in Fig. 10 with the simulated step responses of $R_{d 1}$ and $R_{d 2}$ for reference. We evaluate sensitivity of the step-response to a known change in the haptic device dynamics by removing part of the handwheel from our device. System identification shows that this modification reduces the rotational inertia by approximately $30 \%$. Step responses of the rendered virtual environments using the modified hardware are the dashed traces shown in Fig. 10. We have scaled the applied step torque to produce $180^{\circ} \mathrm{DC}$ response of the virtual environments. 
(a) $P, R_{d 1}$, and $R_{d 2}$

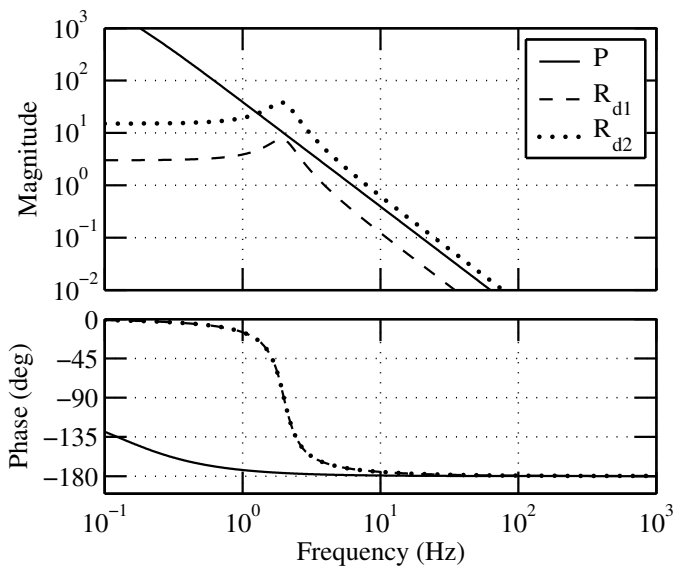

(b) $\Gamma$
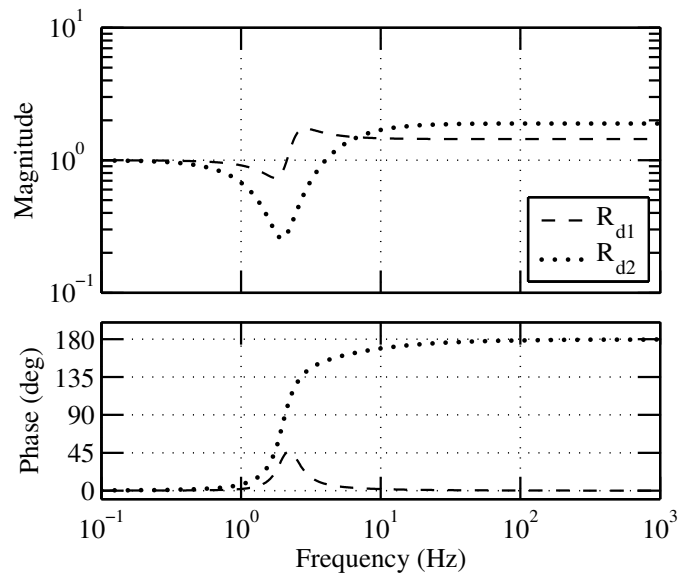

Fig. 6. Predicted tradeoff severity. The frequency responses of $R_{d 1}$ and $R_{d 2}$ relative to the haptic device $P$ are shown in (a). The resulting tradeoff severities $\Gamma$ are shown in (b). As indicated by $\Gamma \approx 1$, no tradeoff exists at low frequencies where $|P|$ is much larger than $\left|R_{d 1}\right|$ or $\left|R_{d 2}\right|$. The most severe tradeoff between performance and sensitivity occurs for $R_{d 2}$ near $2 \mathrm{~Hz}$. At this frequency, $\Gamma$ approaches 0 as $\left|R_{d 2}\right|$ significantly exceeds $|P|$. Note that phase also provides important information; where $|\Gamma|$ crosses 1 near $4-5 \mathrm{~Hz}$, the phase plot indicates that the complex value of $\Gamma$ is not in fact close to 1 , and thus a significant tradeoff still exists.

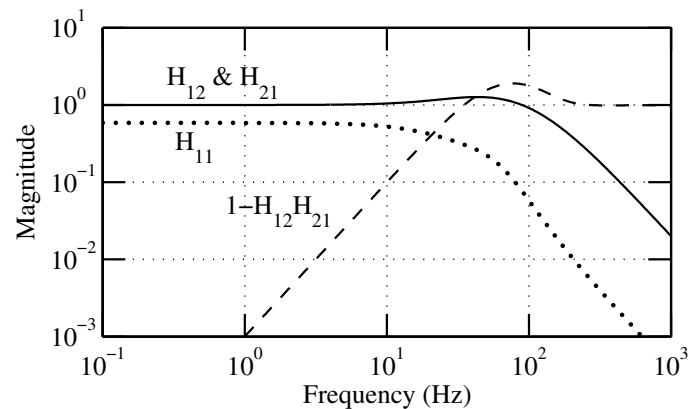

Fig. 7. Generalized virtual coupler design parameters $H_{11}, H_{12}$ and $H_{21}$. As given by (21), distortion $\Theta_{c}$ is bounded above according to $\left|\Theta_{c}\right| \leq\left|H_{11}\right| /\left|R_{d}\right|+$ $\left|H_{12} H_{21}-1\right|$.

As predicted by $\Gamma$ shown in Fig. $6 \mathrm{~b}$, rendering $R_{d 2}$ with low distortion near $2 \mathrm{~Hz}$ involves a more severe tradeoff between transparency and sensitivity than does $R_{d 1}$. For both $R_{d 1}$ and $R_{d 2}$, the peak in $S$ at $2.1 \mathrm{~Hz}$ is a direct consequence of the limit (14). For $R_{d 1}$, the cost of low distortion near $2 \mathrm{~Hz}$ is a peak magnitude in $S$ of 0.8 ; whereas for $R_{d 2}$, the cost of low distortion near $2 \mathrm{~Hz}$ induces a peak in the Bode sensitivity function of 3.9.

Experimental step-responses of $R_{d 1}$ and $R_{d 2}$ as rendered by the cancellation coupler closely match the desired simulated step-responses of $R_{d 1}$ and $R_{d 2}$; however, the step-response of $R_{d 2}$ on the modified haptic device reveals a much greater sensitivity to the hardware. Thus, using a well-tuned controller and the unperturbed device dynamics, we can achieve low distortion for both $R_{d 1}$ and $R_{d 2}$; however larger sensitivity induced to render $R_{d 2}$ causes greater error between the actual response and the desired response given the same variation in the device dynamics.

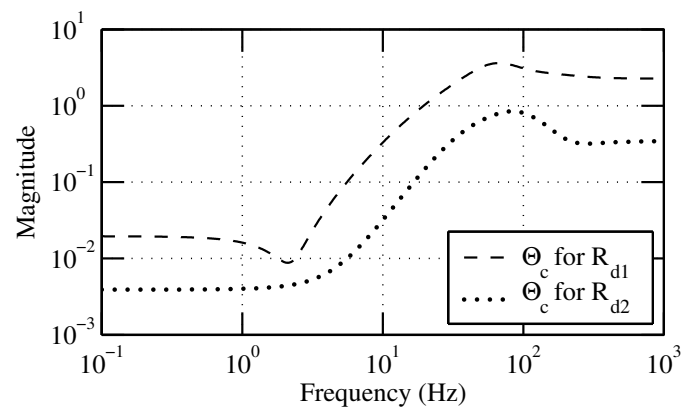

Fig. 8. Predicted closed-loop distortion $\Theta_{c}$ for the cancellation coupler rendering $R_{d 1}$ and $R_{d 2}$.

\section{DISCUSSION}

In this paper we have revealed a fundamental design tradeoff between low distortion rendering of virtual environments and sensitivity of closed-loop responses to parameter variations in the haptic device and model uncertainty. The limit (14) quantifies the cost in terms of sensitivity to attenuate distortion and cannot be circumvent by feedback design. However, reduction of the inherent dynamics of the haptic device, such as reducing damping and inertia, will reduce the sensitivity required to render a virtual environment.

As a practical matter, the range virtual environments that can be rendered well with a particular haptic device is limited. As the magnitude of the virtual environment dynamics $R_{d}$ increase relative to the haptic device dynamics $P$, error in the model of the haptic device $P$ must be reduced to maintain low distortion in the face of high sensitivity. However, the accuracy of available models for a haptic device is typically limited and the hardware dynamics are themselves subject to some variation over time.

The algebraic tradeoff between performance and sensitivity 
(a) $S$ for $R_{d 1}$

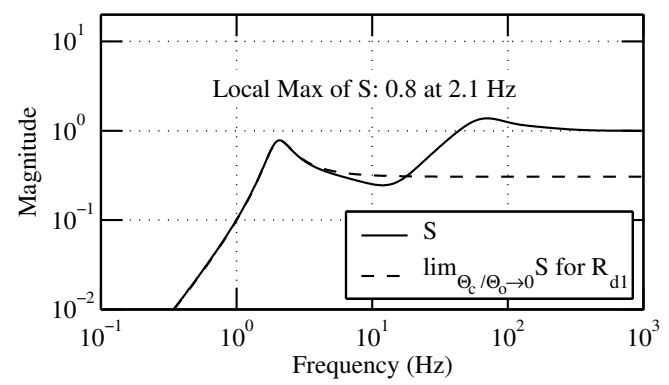

(b) $S$ for $R_{d 2}$

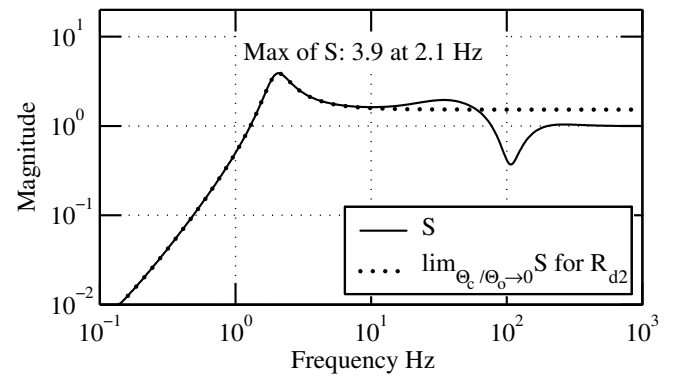

Fig. 9. Predicted Bode sensitivity function $S$ for the cancellation coupler rendering $R_{d 1}$ and $R_{d 2}$, and the limit of $S$ as distortion $\Theta_{c}$ approaches 0 for each virtual environment.

\section{EXPERIMENTAL RESULTS}

(a) Rendered $R_{d 1}$

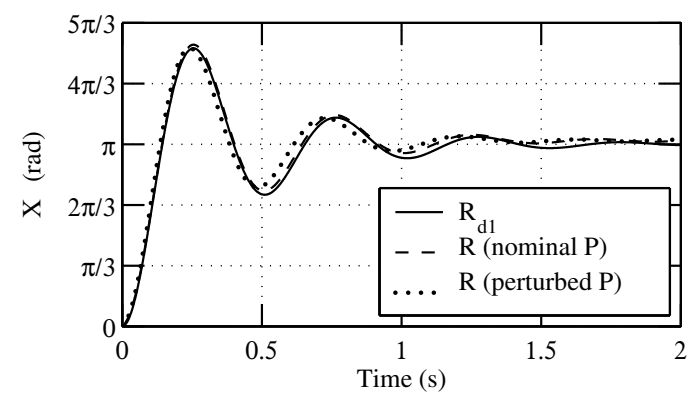

(b) Rendered $R_{d 2}$

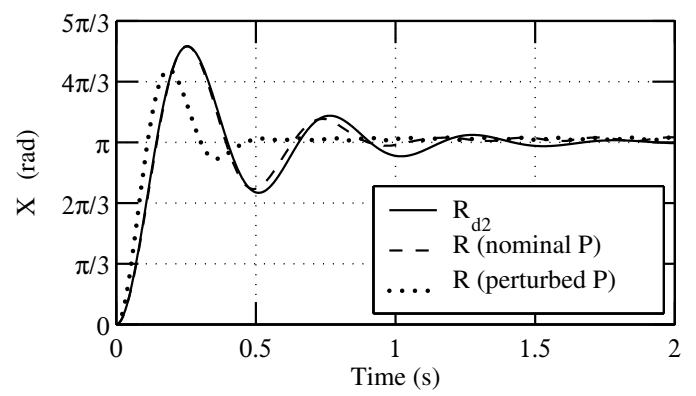

Fig. 10. Experimentally determined and desired step responses of virtual environments $R_{d 1}$ and $R_{d 2}$. Dashed traces are step-responses rendered by the cancellation coupler on a haptic device with dynamics given by (24). Dotted traces are step-responses rendered on a modified device with $30 \%$ less rotational inertia. to hardware dynamics assumes a control architecture with position feedback. Additional sensors can mitigate the tradeoffs inherent to the position feedback architecture. A logical choice is the addition of a force sensor measuring the human operator's force $F$ on the haptic device. With this measurement we may compute the desired position $X_{d}$ by $R_{d} F$. Then typical high-gain control techniques, not subject to the algebraic tradeoff between performance and sensitivity, could be applied to make the haptic device position $X$ track $X_{d}$.

\section{ACKNOWLEDGMENT}

This research was supported by the National Science Foundation under Grant EHS-0410553.

\section{REFERENCES}

[1] J. E. Colgate, M. C. Stanley, and J. M. Brown, "Issues in the haptic display of tool use," in Proc. IEEE/RSJ Int'l Conf. on Intelligent Robots and Control, Pittsburgh, PA, 1995, pp. 140-45.

[2] J. E. Colgate and G. G. Schenkel, "Passivity of a class of sampled-data systems: application to haptic interfaces," J. of Rob. Sys., vol. 14, no. 1, pp. 37-47, Jan. 1997.

[3] M. Minsky, M. Ouh-Young, O. Steele, F. P. Brooks, and M. Behensky, "Feeling and seeing: Issues in forces in display," in Proc. Symp. on Interact. 3D Graphics, vol. 24, no. 2, Snowbird, UT, 1990, pp. 235-41.

[4] M. Mahvash and V. Hayward, "High-fidelity passive force-reflecting virtual environments," IEEE Trans. Robot., vol. 21, no. 1, pp. 38-46, Feb. 2005.

[5] J. S. Mehling, J. E. Colgate, and M. A. Peshkin, "Increasing the impedance range of haptic display by adding electrical damping," in $1^{\text {st }}$ Joint Eurohaptics Conf. \& Symp. on Haptic Interfaces for Virtual Environments and Teleoperator Systems, Pisa, Italy, 2005, pp. 257-62.

[6] N. Diolaiti, G. Niemeyer, F. Barbagli, and J. K. J. Salisbury, "Stability of haptic rendering: Discretization, quantization, time delay, and Coulomb effects," IEEE Trans. Robot., vol. 22, no. 2, pp. 256-68, Apr. 2006.

[7] X. Shen and M. Goldfarb, "On the enhanced passivity of pneumatically actuated impedance-type haptic interfaces," IEEE Trans. Robot., vol. 22, no. 3, pp. 470-80, June 2006.

[8] M. C. Çavuşoğlu, D. Feygin, and F. Tendick, "A critical study of the mechanical and electrical properties of the PHANToM haptic interface and improvements for high-performance control," Presence, vol. 11, no. 6, pp. 555-68, Dec. 2002.

[9] J. J. Abbott and A. M. Okamura, "Effects of position quantization and sampling rate on virtual-wall passivity," IEEE Trans. Robot., vol. 21, no. 5, pp. 952-64, Oct. 2005.

[10] B. E. Miller, J. E. Colgate, and R. A. Freeman, "Guarenteed stability of haptic systems with nonlinear virtual environments," IEEE Trans. on Robot. \& Automat., vol. 16, no. 6, pp. 712-19, Dec. 2000.

[11] M. Fardad and B. Bamieh, "A frequency domain analysis and synthesis of the passivity of sampled-data systems," in Proc. $43^{\text {rd }}$ IEEE Conf. on Decision and Contr., vol. 3, Nassau, Bahamas, 2004, pp. 2358-63.

[12] S. Skogestad and I. Postlethwaite, Multivariable Feedback Control: Analysis and Design. New York: Wiley, 1997.

[13] J. S. Freudenberg, C. V. Hollot, R. H. Middleton, and V. Toochinda, "Fundamental design limitations of the general control configuration," IEEE Tran. Autom. Contr., vol. 48, no. 8, pp. 1355-70, Aug. 2003.

[14] D. A. Lawrence, "Stability and transparency in bilateral teleoperation," IEEE Trans. on Robot. \& Automat., vol. 9, no. 5, pp. 624-37, Oct. 1993.

[15] R. J. Adams and B. Hannaford, "Stable haptic interaction with virtual environments," IEEE Trans. on Robot. \& Automat., vol. 15, no. 3, pp. 465-74, June 1999.

[16] R. B. Gillespie, M. B. Hoffman, and J. Freudenberg, "Haptic interface for hands-on instruction in system dynamics and embedded control," in Proc. Symp. on Haptic Interfaces for Virtual Environment and Teleoperator Systems, Los Angeles, CA, 2003, pp. 410-15. 